\title{
Lefschetz fixed point theorem for digital images
}

\section{Ozgur Ege ${ }^{1 *}$ and Ismet Karaca ${ }^{2}$}

"Correspondence: ozgur.ege@cbu.edu.tr

${ }^{1}$ Department of Mathematics, Celal Bayar University, Muradiye, Manisa 45140, Turkey

Full list of author information is available at the end of the article

\section{严 Springer}

\begin{abstract}
In this article we study the fixed point properties of digital images. Moreover, we prove the Lefschetz fixed point theorem for a digital image. We then give some examples about the fixed point property. We conclude that sphere-like digital images have the fixed point property.

MSC: 55N35; 68R10; 68U05; 68U10

Keywords: digital image; Lefschetz fixed point theorem; Euler characteristic
\end{abstract}

\section{Introduction}

Digital topology is important for computer vision, image processing and computer graphics. In this area many researchers, such as Rosenfeld, Kong, Kopperman, Kovalevsky, Boxer, Karaca, Han and others, have characterized the properties of digital images with tools from topology, especially algebraic topology.

The homology theory is the major subject of algebraic topology. It is used in the classification problems of topological spaces. The simplicial homology is one of the homology theories. Homology groups are topological invariants which are related to the different $n$ dimensional holes, connected components of a geometric object. For example, the torus contains one 0 -dimensional hole, two 1-dimensional holes and one 2-dimensional hole.

The Lefschetz fixed point theorem is a formula that counts fixed points of a continuous mapping from a compact topological space $X$ to itself by means of traces of the induced mappings on the homology groups of $X$. In 1926, Lefschetz introduced the Lefschetz number of a map and proved that if the number is nonzero, then the map has a fixed point. Since the Lefschetz number is defined in terms of the homology homomorphism induced by the map, it is a homotopy invariant. As a result, a nonzero Lefschetz number implies that each of the maps in the given homotopy class has at least one fixed point. The Lefschetz fixed point theorem generalizes a collection of fixed point theorems for different topological spaces. We will work entirely with digital images that are digital simplicial complexes or retracts of digital simplicial complexes.

Arslan et al. [1] introduced the simplicial homology groups of $n$-dimensional digital images from algebraic topology. They also computed simplicial homology groups of $M_{S S_{18}}$. Boxer et al. [2] expanded the knowledge of simplicial homology groups of digital images. They studied the simplicial homology groups of certain minimal simple closed surfaces, extended an earlier definition of the Euler characteristics of a digital image, and computed the Euler characteristic of several digital surfaces. Demir and Karaca [3] computed

( 2013 Ege and Karaca; licensee Springer. This is an Open Access article distributed under the terms of the Creative Commons Attribution License (http://creativecommons.org/licenses/by/2.0), which permits unrestricted use, distribution, and reproduction in any medium, provided the original work is properly cited. 
the simplicial homology groups of some digital surfaces such as $M S S_{18} \sharp M S S_{18}, M S S_{6}$ and $M S_{6} \sharp M S S_{6}$.

Karaca and Ege [4] studied some results related to the simplicial homology groups of $2 D$ digital images. They showed that if a bounded digital image $X \subset \mathbb{Z}$ is nonempty and $\kappa$-connected, then its homology groups at the first dimension are a trivial group. They also proved that the homology groups of the operands of a wedge of digital images need not be additive. Ege and Karaca [5] gave characteristic properties of the simplicial homology groups of digital images and then investigated the Eilenberg-Steenrod axioms for the simplicial homology groups of digital images.

This paper is organized as follows. The second section provides the general notions of digital images with $\kappa$-adjacency relations, digital homotopy groups and digital homology groups. In Section 3 we present the Lefschetz fixed point theorem for digital images and provide some of its important applications. In the last section we make some conclusions about this topic.

\section{Preliminaries}

Let $\mathbb{Z}$ be the set of integers. $A$ (binary) digital image is a pair $(X, \kappa)$, where $X \subset \mathbb{Z}^{n}$ for some positive integer $n$ and $\kappa$ represents certain adjacency relation for the members of $X$. A variety of adjacency relations are used in the study of digital images. We give one of them. Let $l, n$ be positive integers, $1 \leq l \leq n$ and two distinct points $p=\left(p_{1}, p_{2}, \ldots, p_{n}\right)$, $q=\left(q_{1}, q_{2}, \ldots, q_{n}\right)$ in $\mathbb{Z}^{n}, p$ and $q$ are $k_{l}$-adjacent [6] if there are at most $l$ distinct coordinates $j$, for which $\left|p_{j}-q_{j}\right|=1$ and for all other coordinates $j, p_{j}=q_{j}$. The number of points $q \in \mathbb{Z}^{n}$ that are adjacent to a given point $p \in \mathbb{Z}^{n}$ is represented by a $k_{l}$-adjacency relation. From this viewpoint, the $k_{1}$-adjacency on $\mathbb{Z}$ is denoted by the number 2 and $k_{1}$-adjacent points are called 2-adjacent. In a similar way, we call 4-adjacent and 8-adjacent for $k_{1}$ and $k_{2}$-adjacent points of $\mathbb{Z}^{2}$; and in $\mathbb{Z}^{3}, 6$-adjacent, 18-adjacent and 26-adjacent for $k_{1}, k_{2}$ and $k_{3}$-adjacent points, respectively.

A $\kappa$-neighbor of $p \in \mathbb{Z}^{n}$ is a point of $\mathbb{Z}^{n}$ which is $\kappa$-adjacent to $p$, where $\kappa$ is an adjacency relation defined on $\mathbb{Z}^{n}$. A digital image $X \subset \mathbb{Z}^{n}$ is $\kappa$-connected [7] if and only if for every pair of different points $x, y \in X$, there is a set $\left\{x_{0}, x_{1}, \ldots, x_{r}\right\}$ of points of a digital image $X$ such that $x=x_{0}, y=x_{r}$ and $x_{i}$ and $x_{i+1}$ are $\kappa$-neighbors where $i=0,1, \ldots, r-1$. A maximal $\kappa$-connected subset of a digital image $X$ is called $a \kappa$-component of $X$. A set of the form

$$
[a, b]_{\mathbb{Z}}=\{z \in \mathbb{Z} \mid a \leq z \leq b\}
$$

is said to be $a$ digital interval [8], where $a, b \in \mathbb{Z}$ with $a<b$.

Let $\left(X, \kappa_{0}\right) \subset \mathbb{Z}^{n_{0}}$ and $\left(Y, \kappa_{1}\right) \subset \mathbb{Z}^{n_{1}}$ be digital images. A function $f: X \longrightarrow Y$ is called $\left(\kappa_{0}, \kappa_{1}\right)$-continuous [9] if for every $\kappa_{0}$-connected subset $U$ of $X, f(U)$ is a $\kappa_{1}$-connected subset of $Y$.

In a digital image $X$, if there is a $(2, \kappa)$-continuous function $f:[0, m]_{\mathbb{Z}} \rightarrow X$ such that $f(0)=x$ and $f(m)=y$, then we say that there exists $a$ digital $\kappa$-path [10] from $x$ to $y$. If $f(0)=f(m)$, then $f$ is called digital $\kappa$-loop and the point $f(0)$ is the base point of the loop $f$. When a digital loop $f$ is a constant function, it is said to be a trivial loop. A simple closed $\kappa$-curve of $m \geq 4$ points in a digital image $X$ is a sequence $\{f(0), f(1), \ldots, f(m-1)\}$ of images of the $\kappa$-path $f:[0, m-1]_{\mathbb{Z}} \longrightarrow X$ such that $f(i)$ and $f(j)$ are $\kappa$-adjacent if and only if $j=$ $i \pm \bmod m$. 
Definition 2.1 Let $\left(X, \kappa_{0}\right) \subset \mathbb{Z}^{n_{0}}$ and $\left(Y, \kappa_{1}\right) \subset \mathbb{Z}^{n_{1}}$ be digital images. A function $f: X \longrightarrow$ $Y$ is $a\left(\kappa_{0}, \kappa_{1}\right)$-isomorphism [1] if $f$ is $\left(\kappa_{0}, \kappa_{1}\right)$-continuous and bijective and $f^{-1}: Y \rightarrow X$ is $\left(\kappa_{1}, \kappa_{0}\right)$-continuous and it is denoted by $X \approx_{\left(\kappa_{0}, \kappa_{1}\right)} Y$.

For a digital image $(X, \kappa)$ and its subset $(A, \kappa)$, we call $(X, A)$ a digital image pair with $\kappa$-adjacency. If $A$ is a singleton set $\left\{x_{0}\right\}$, then $\left(X, x_{0}\right)$ is called a pointed digital image.

Definition 2.2 [9] Let $\left(X, \kappa_{0}\right) \subset \mathbb{Z}^{n_{0}}$ and $\left(Y, \kappa_{1}\right) \subset \mathbb{Z}^{n_{1}}$ be digital images. Two $\left(\kappa_{0}, \kappa_{1}\right)$ continuous functions $f, g: X \longrightarrow Y$ are said to be digitally $\left(\kappa_{0}, \kappa_{1}\right)$-homotopic in $Y$ if there is a positive integer $m$ and a function $H: X \times[0, m]_{\mathbb{Z}} \longrightarrow Y$ such that

- for all $x \in X, H(x, 0)=f(x)$ and $H(x, m)=g(x)$;

- for all $x \in X$, the induced function $H_{x}:[0, m]_{\mathbb{Z}} \longrightarrow Y$ defined by

$$
H_{x}(t)=H(x, t) \quad \text { for all } t \in[0, m]_{\mathbb{Z}}
$$

is $\left(2, \kappa_{1}\right)$-continuous; and

- for all $t \in[0, m]_{\mathbb{Z}}$, the induced function $H_{t}: X \longrightarrow Y$ defined by

$$
H_{t}(x)=H(x, t) \quad \text { for all } x \in X
$$

is $\left(\kappa_{0}, \kappa_{1}\right)$-continuous.

The function $H$ is called a digital $\left(\kappa_{0}, \kappa_{1}\right)$-homotopy between $f$ and $g$. If these functions are digitally $\left(\kappa_{0}, \kappa_{1}\right)$-homotopic, this is denoted by $f \simeq_{\kappa_{0}, \kappa_{1}} g$. The digital $\left(\kappa_{0}, \kappa_{1}\right)$-homotopy relation [9] is equivalence among digitally continuous functions $f:\left(X, \kappa_{0}\right) \longrightarrow\left(Y, \kappa_{1}\right)$.

Definition 2.3 [9] (i) A digital image $(X, \kappa)$ is said to be $\kappa$-contractible if its identity map is $(\kappa, \kappa)$-homotopic to a constant function $\bar{c}$ for some $c \in X$, where the constant function $\bar{c}: X \longrightarrow X$ is defined by $\bar{c}(x)=c$ for all $x \in X$.

(ii) Let $(X, A)$ be a digital image pair with $\kappa$-adjacency. Let $i: A \longrightarrow X$ be the inclusion function. $A$ is called a $\kappa$-retract of $X$ if and only if there is a $\kappa$-continuous function $r: X \longrightarrow$ $A$ such that $r(a)=a$ for all $a \in A$. Then the function $r$ is called a $\kappa$-retraction of $X$ onto $A$.

(iii) A digital homotopy $H: X \times[0, m]_{\mathbb{Z}} \longrightarrow X$ is a deformation retract [11] if the induced map $H(-, 0)$ is the identity map $1_{X}$ and the induced map $H(-, m)$ is retraction of $X$ onto $H(X \times\{m\}) \subset X$. The set $H(X \times\{m\})$ is called a deformation retract of $X$.

Definition 2.4 [12] Let $S$ be a set of nonempty subsets of a digital image $(X, \kappa)$. Then the members of $S$ are called simplexes of $(X, \kappa)$ if the following hold:

(a) If $p$ and $q$ are distinct points of $s \in S$, then $p$ and $q$ are $\kappa$-adjacent.

(b) If $s \in S$ and $\emptyset \neq t \subset s$, then $t \in S$.

An $m$-simplex is a simplex $S$ such that $|S|=m+1$.

Let $P$ be a digital $m$-simplex. If $P^{\prime}$ is a nonempty proper subset of $P$, then $P^{\prime}$ is called a face of $P$. We write $\operatorname{Vert}(P)$ to denote the vertex set of $P$, namely the set of all digital 0 -simplexes in $P$. A digital subcomplex $A$ of a digital simplicial complex $X$ with $\kappa$-adjacency is a digital simplicial complex [12] contained in $X$ with $\operatorname{Vert}(A) \subset \operatorname{Vert}(X)$. 
Definition 2.5 Let $(X, \kappa)$ be a finite collection of digital $m$-simplexes, $0 \leq m \leq d$ for some nonnegative integer $d$. If the following statements hold, then $(X, \kappa)$ is called [1] a finite digital simplicial complex:

(1) If $P$ belongs to $X$, then every face of $P$ also belongs to $X$.

(2) If $P, Q \in X$, then $P \cap Q$ is either empty or a common face of $P$ and $Q$.

The dimension of a digital simplicial complex $X$ is the largest integer $m$ such that $X$ has an $m$-simplex. $C_{q}^{\kappa}(X)$ is a free abelian group [1] with basis of all digital $(\kappa, q)$-simplexes in $X$.

Corollary 2.6 [1] Let $(X, \kappa) \subset \mathbb{Z}^{n}$ be a digital simplicial complex of dimension $m$. Then, for all $q>m, C_{q}^{\kappa}(X)$ is a trivial group.

Let $(X, \kappa) \subset \mathbb{Z}^{n}$ be a digital simplicial complex of dimension $m$. The homomorphism $\partial_{q}: C_{q}^{\kappa}(X) \longrightarrow C_{q-1}^{\kappa}(X)$ defined (see [1]) by

$$
\partial_{q}\left(\left\langle p_{0}, p_{1}, \ldots, p_{q}\right\rangle\right)= \begin{cases}\sum_{i=0}^{q}(-1)^{i}\left\langle p_{0}, p_{1}, \ldots, \hat{p}_{i}, \ldots, p_{q}\right\rangle, & q \leq m, \\ 0, & q>m\end{cases}
$$

is called a boundary homomorphism, where $\hat{p}_{i}$ means delete the point $p_{i}$. In [1], it is shown that for all $1 \leq q \leq m$,

$$
\partial_{q-1} \circ \partial_{q}=0 .
$$

Definition 2.7 [2] Let $(X, \kappa)$ be a digital simplicial complex.

(1) $Z_{q}^{\kappa}(X)=\operatorname{Ker} \partial_{q}$ is called the group of digital simplicial $q$-cycles.

(2) $B_{q}^{\kappa}(X)=\operatorname{Im} \partial_{q+1}$ is called the group of digital simplicial $q$-boundaries.

(3) $H_{q}^{\kappa}(X)=Z_{q}^{\kappa}(X) / B_{q}^{\kappa}(X)$ is called the $q$ th digital simplicial homology group.

We recall some important examples about digital homology groups of certain digital images.

Example 2.8 Let $X=\{(0,0),(1,0),(0,1),(1,1)\} \subset \mathbb{Z}^{2}$ be a digital image with 4-adjacency. In [1], it is shown that digital homology groups of $X$ are:

$$
H_{q}^{4}(X)= \begin{cases}\mathbb{Z}, & q=0 \\ 0, & q \neq 0\end{cases}
$$

Example 2.9 Let $X=\{(0,1),(0,0),(1,1)\} \subset \mathbb{Z}^{2}$ be a digital image with 8 -adjacency. Havana et al. [1] showed that digital homology groups of $(X, 8)$ are

$$
H_{q}^{8}(X)= \begin{cases}\mathbb{Z}, & q=0 \\ 0, & q \neq 0\end{cases}
$$



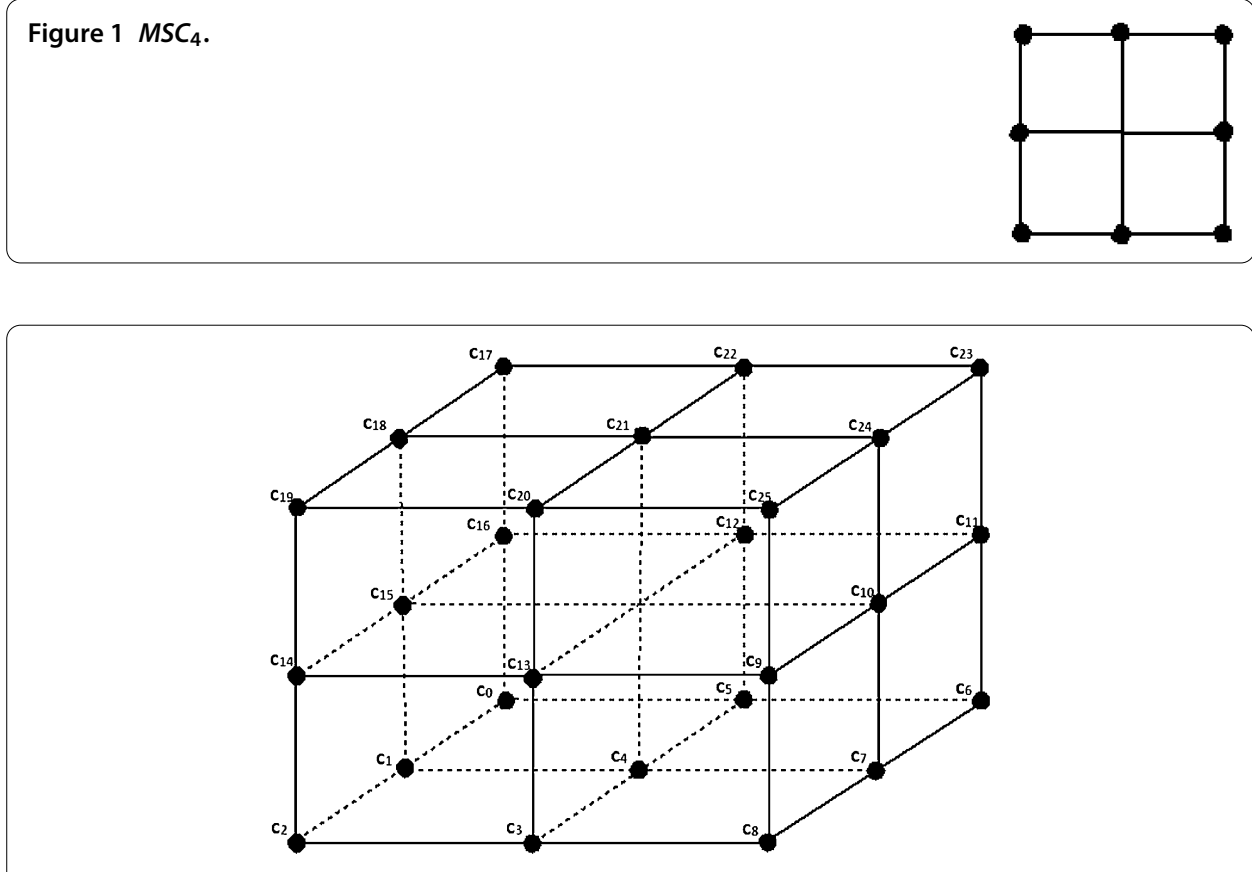

Figure $2 \mathrm{MSS}_{6}$.

Example 2.10 $\mathrm{MSC}_{4}=\{(1,0),(1,-1),(1,1),(0,1),(0,-1),(-1,1),(-1,0),(-1,-1)\}$ is a digital image with 4 -adjacency in $\mathbb{Z}^{2}$ (see Figure 1). The following result is given in [1].

$$
H_{q}^{4}\left(M S C_{4}\right)= \begin{cases}\mathbb{Z}, & q=0,1, \\ 0, & q \neq 0,1 .\end{cases}
$$

Example 2.11 $\mathrm{MSS}_{6}$ is a digital image with 6-adjacency in $\mathbb{Z}^{3}$ (see Figure 2). In [3] Demir and Karaca showed that the digital homology groups of $M_{S S}$ are as follows:

$$
H_{q}^{6}\left(M S S_{6}\right)= \begin{cases}\mathbb{Z}, & q=0, \\ \mathbb{Z}^{23}, & q=1, \\ 0, & q \neq 0,1 .\end{cases}
$$

Example 2.12 Let $M S S_{6}^{\prime}=\left\{c_{0}=(0,0,0), c_{1}=(1,0,0), c_{2}=(1,1,0), c_{3}=(0,1,0), c_{4}=(0\right.$, $\left.0,1), c_{5}=(1,0,1), c_{6}=(1,1,1), c_{7}=(0,1,1)\right\}$ be a digital image with 6 -adjacency (see Figure 3). In [10], it is shown that

$$
H_{q}^{6}\left(M_{6}^{\prime}\right)= \begin{cases}\mathbb{Z}, & q=0 \\ \mathbb{Z}^{5}, & q=1 \\ 0, & q \neq 0,1\end{cases}
$$

Theorem 2.13 [2] Let $(X, \kappa)$ be a directed digital simplicial complex of dimension $m$. 


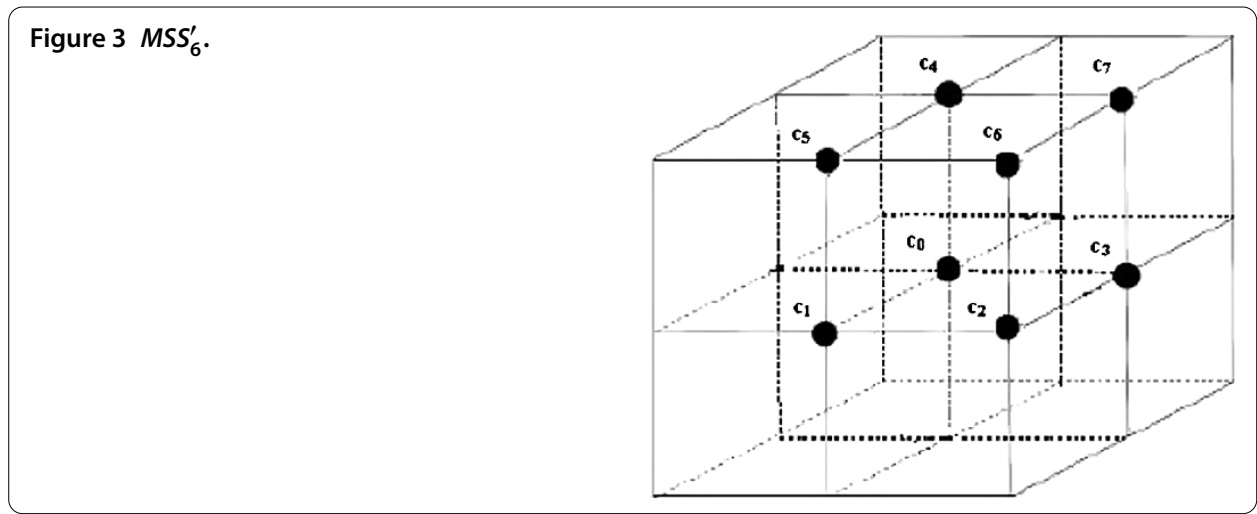

(1) $H_{q}^{k}(X)$ is a finitely generated abelian group for every $q \geq 0$.

(2) $H_{q}^{\kappa}(X)$ is a trivial group for all $q>m$.

(3) $H_{m}^{\kappa}(X)$ is a free abelian group, possible zero.

In [2], it is proven that for each $q \geq 0, H_{q}^{\kappa}$ is a covariant functor from the category of digital simplicial complexes and simplicial maps to the category of abelian groups.

Definition 2.14 [2] Let $f:\left(X, \kappa_{0}\right) \longrightarrow\left(Y, \kappa_{1}\right)$ be a function between two digital images. If for every digital $\left(\kappa_{0}, m\right)$-simplex $P$ determined by $\kappa_{0}$ in $X, f(P)$ is a $\left(\kappa_{1}, n\right)$-simplex in $Y$ for some $n \leq m$, then $f$ is called a digital simplicial map.

For a digital simplicial map $f:\left(X, \kappa_{0}\right) \longrightarrow\left(Y, \kappa_{1}\right)$ and $q \geq 0$, two induced homomorphisms $f_{\#}: C_{q}^{\kappa_{0}}(X) \longrightarrow C_{q}^{\kappa_{1}}(Y)$ and $f_{*}: H_{q}^{\kappa_{0}}(X) \longrightarrow H_{q}^{\kappa_{1}}(Y)$ are defined by (see [2])

$$
\begin{aligned}
& f_{\#}\left(\left\langle p_{0}, \ldots, p_{q}\right\rangle\right)=\left\langle f_{\#}\left(p_{0}\right), \ldots, f_{\#}\left(p_{q}\right)\right\rangle, \\
& f_{*}\left(z+B_{q}^{\kappa}(X)\right)=f_{\#}(z)+B_{q}^{\kappa}(Y),
\end{aligned}
$$

where $z \in Z_{q}^{\kappa}(X)$, respectively.

Definition 2.15 [10] Let $X$ and $Y$ be digital images in $\mathbb{Z}^{n}$, using the same adjacency notion, denoted by $\kappa$, such that $X \cap Y=\left\{x_{0}\right\}$, where $x_{0}$ is the only point of $X$ adjacent to any point of $Y$ and $x_{0}$ is the only point of $Y$ adjacent to any point of $X$. Then the wedge of $X$ and $Y$, denoted by $X \wedge Y$, is the image $X \wedge Y=X \cup Y$, with $\kappa$-adjacency.

\section{The Lefschetz fixed point theorem for digital images}

A digital image $(X, \kappa)$ is said to have the fixed point property if for any $(\kappa, \kappa)$-continuous function $f:(X, \kappa) \longrightarrow(X, \kappa)$, there exists $x \in X$ such that $f(x)=x$. The fixed point property is a topological invariant, i.e., is preserved by any digital isomorphism. The fixed point property is also preserved by any retraction. The Lefschetz fixed point theorem determines when there exist fixed points of a map on a finite digital simplicial complex using a characteristic of the map known as the Lefschetz number.

Definition 3.1 For a map $f:(X, \kappa) \longrightarrow(X, \kappa)$, where $(X, \kappa)$ is a digital image whose digital homology groups are finitely generated and vanish above some dimension, the Lefschetz 
number $\lambda(f)$ is defined as follows:

$$
\lambda(f)=\sum_{i=0}^{n}(-1)^{i} \operatorname{tr}\left(f_{*}\right)
$$

where $f_{*}: H_{i}^{\kappa}(X) \longrightarrow H_{i}^{\kappa}(X)$.

Theorem 3.2 Let $G$ be a free abelian group, and let $1_{G}: G \rightarrow G$ be the identity homomorphism. Then $\operatorname{tr}\left(1_{G}\right)=\operatorname{rank}(G)$, where $\operatorname{tr}\left(1_{G}\right)$ is the trace of $1_{G}$.

Proof Since $G$ is a free abelian group, the $\operatorname{rank}$ of $G$ is 1 ; i.e., we have $\operatorname{rank}(G)=1$. Moreover, the trace of $1_{G}$ is 1 because $1_{G}: G \longrightarrow G$ is the identity map and has an identity $1 \times 1$-matrix [1]. So, we have $\operatorname{rank}(G)=\operatorname{tr}\left(1_{G}\right)$.

Theorem 3.3 If $(X, \kappa)$ is a finite digital simplicial complex, or the retract of some finite digital simplicial complex, and $f:(X, \kappa) \longrightarrow(X, \kappa)$ is a map with $\lambda(f) \neq 0$, then $f$ has a fixed point.

Proof Suppose that $f$ has no fixed points. Then we must show that $\lambda(f)=0$. The approach would be to show that all $\operatorname{tr}\left(f_{*}: H_{i}^{\kappa}(X) \longrightarrow H_{i}^{\kappa}(X)\right)$ are zero. For this purpose, we need the following which is called the Lefschetz principle:

$$
\lambda(f)=\sum_{i=0}^{n}(-1)^{i} \operatorname{tr}\left(f_{\sharp}: C_{i}^{\kappa}(X) \longrightarrow C_{i}^{\kappa}(X)\right) .
$$

To compute the digital homology groups of $(X, \kappa)$, we use digital simplicial chains $C_{i}^{\kappa}(X)$. Since $f$ has no fixed points and $(X, \kappa)$ is compact, $f$ must move points at least a fixed positive distance, say $\delta$. Then we use simplexes which have diameter less than $\delta / 10$, and we approximate $f$ by a digital simplicial map $g$ so that pointwise $g$ is within $\delta / 2$ of $f$. We may assume that $\delta$ is so small that $g$ is homotopic to $f$, so we may as well assume that $g=f$. Because $f$ moves points at least $\delta$ and our digital simplexes are at most $\delta / 10$ in diameter, it is impossible for $f$ to map any digital simplex to itself, so it is immediate that for all $k$,

$$
\operatorname{tr}\left(f_{\#}: C_{i}^{\kappa}(X) \longrightarrow C_{i}^{\kappa}(X)\right)=0 .
$$

Theorem 3.4 (One-dimensional Brouwer fixed point theorem) Every (2,2)-continuous function $f:[0,1]_{\mathbb{Z}} \longrightarrow[0,1]_{\mathbb{Z}}$ has a fixed point.

Proof Let $f:[0,1]_{\mathbb{Z}} \longrightarrow[0,1]_{\mathbb{Z}}$ be $(2,2)$-continuous. Since $f(0)=0$ and $f(1)=1, f$ has a fixed point.

Theorem 3.5 (Two-dimensional Brouwer fixed point theorem) Let $X=\{(0,0),(1,0),(0,1)$, $(1,1)\} \subset \mathbb{Z}^{2}$ be a digital image with 4-adjacency. Every $(4,4)$-continuous function $f$ : $(X, 4) \longrightarrow(X, 4)$ has a fixed point.

Proof We compute the Lefschetz number of $X$. Let $f:(X, 4) \longrightarrow(X, 4)$. Take the induced maps $f_{*}: H_{q}^{4}(X) \longrightarrow H_{q}^{4}(X)$. For $q=0$, we have $f_{*}: H_{0}^{4}(X) \longrightarrow H_{0}^{4}(X)$; i.e., $f_{*}: \mathbb{Z} \longrightarrow \mathbb{Z}$ is 
identity homomorphism. By Theorem 3.2,

$$
\operatorname{tr}\left(f_{*}\right)=\operatorname{rank}(\mathbb{Z})=1
$$

For all $q>0$, it follows from Example 2.8 that $f_{*}$ is a zero map. Thus we have

$$
\begin{aligned}
\lambda(f) & =\sum_{i=0}^{n}(-1)^{i} \operatorname{tr}\left(f_{*}\right) \\
& =1-0+0-0+\cdots \\
& =1 .
\end{aligned}
$$

Since $\lambda(f)=1 \neq 0$, Theorem 3.3 implies that $f$ has a fixed point. As a result, twodimensional Brouwer fixed point theorem holds for digital images.

We conclude now by showing that the fixed point property is a topological property; i.e., two topological spaces which are topologically equivalent to each other either both have or both lack the fixed point property. Because one of the main goals of topology is to discover what properties are maintained when moving between topologically equivalent spaces, this is an incredibly important proposition to make.

Theorem 3.6 Let $(X, \kappa)$ and $\left(Y, \kappa^{\prime}\right)$ be digital images such that $X \approx_{\left(\kappa, \kappa^{\prime}\right)} Y$. If $(X, \kappa)$ has the fixed point property, then $\left(Y, \kappa^{\prime}\right)$ has the fixed point property.

Proof Since $X \approx_{\left(\kappa, \kappa^{\prime}\right)} Y$, there exists a bijective function $f:(X, \kappa) \longrightarrow\left(Y, \kappa^{\prime}\right)$ such that $f$ is $\left(\kappa, \kappa^{\prime}\right)$-continuous and that its inverse $f^{-1}$ is $\left(\kappa^{\prime}, \kappa\right)$-continuous. Also, $X$ has the fixed point property; i.e., every $(\kappa, \kappa)$-continuous function $g:(X, \kappa) \longrightarrow(X, \kappa)$ has a fixed point. Now, let $h:\left(Y, \kappa^{\prime}\right) \longrightarrow\left(Y, \kappa^{\prime}\right)$ be $\left(\kappa^{\prime}, \kappa^{\prime}\right)$-continuous. Then $h \circ f:(X, \kappa) \longrightarrow\left(Y, \kappa^{\prime}\right)$ and, consequently, $f^{-1} \circ h \circ f:(X, \kappa) \longrightarrow(X, \kappa)$ are certainly $\left(\kappa, \kappa^{\prime}\right)$-continuous and $\left(\kappa^{\prime}, \kappa\right)$-continuous, respectively. However, since $X$ has the fixed point property, $f^{-1}(h(f(x)))=x$ for some $x \in X$. It follows that $f\left(f^{-1}(h(f(x)))\right)=f(x)$, which implies that $h(f(x))=f(x)$, proving that $h$ has a fixed point, namely $f(x)$.

Corollary 3.7 The fixed point property is a topological invariant for digital images.

Theorem 3.8 Let $(X, \kappa)$ be any digital image, and let $f:(X, \kappa) \longrightarrow(X, \kappa)$ be any $(\kappa, \kappa)$ continuous map. If $X$ is $\kappa$-contractible, then $f$ has a fixed point.

Proof If $X$ is $\kappa$-contractible, then we have $f \simeq_{\kappa, \kappa} 1_{X}$. So, $X$ and a single point image have the same digital homology groups. Hence we have

$$
H_{i}^{\kappa}(X)= \begin{cases}\mathbb{Z}, & i=0, \\ 0, & i \neq 0\end{cases}
$$

Let $f:(X, \kappa) \longrightarrow(X, \kappa)$. If we take the induced homomorphisms $f_{*}: H_{q}^{\kappa}(X) \longrightarrow H_{q}^{\kappa}(X)$, for $q=0$, we have the identity homomorphism $f_{*}: \mathbb{Z} \longrightarrow \mathbb{Z}$. By Theorem 3.2,

$$
\operatorname{tr}\left(f_{*}\right)=\operatorname{rank}(\mathbb{Z})=1 .
$$


Moreover, for all $q>0$, it follows from Example 2.8 that $f_{*}$ is a zero map. So, the Lefschetz number of $f$ is

$$
\lambda(f)=\sum_{i=0}^{\infty} \operatorname{tr}\left(f_{i}\right)=1-0+0-\cdots=1 .
$$

By Theorem 3.3, we have that $f$ has a fixed point.

Example 3.9 If $X$ is the digital image in Example 2.9, then for any $(8,8)$-continuous map $f:(X, 8) \longrightarrow(X, 8)$, the Lefschetz number $\lambda(f)$ is given by the trace of the induced map on homology

$$
f_{*}: H_{0}^{8}(X)=\mathbb{Z} \longrightarrow H_{0}^{8}(X)=\mathbb{Z}
$$

which is simply the identity function and thus has a nonzero trace. From Theorem 3.3, every map on a digital image $(X, 8)$ has at least one fixed point.

Corollary 3.10 Any digital image $(X, \kappa)$ with the same digital homology groups as a single point image always has a fixed point.

Theorem 3.11 Let $(X, \kappa)$ be a digital image and $(A, \kappa)$ be a digital $\kappa$-retract of $(X, \kappa)$. If $(X, \kappa)$ has the fixed point property, then $(A, \kappa)$ has the fixed point property.

Proof Let $(A, \kappa)$ be a digital $\kappa$-retract of $(X, \kappa)$. Then there exists a digital $\kappa$-retraction map $r$ such that $r \circ i=1_{(A, \kappa)}$, where $i:(A, \kappa) \longrightarrow(X, \kappa)$ is an inclusion map. Since $(X, \kappa)$ has the fixed point property, every $(\kappa, \kappa)$-continuous function $f:(X, \kappa) \longrightarrow(X, \kappa)$ has a fixed point, that is, there exists a point $x \in X$ such that $f(x)=x$. We have

$$
(A, \kappa) \stackrel{i}{\longrightarrow}(X, \kappa) \stackrel{f}{\longrightarrow}(X, \kappa) \stackrel{r}{\longrightarrow}(A, \kappa) .
$$

For all $x \in(A, \kappa)$,

$$
r \circ f \circ i(x)=r \circ f(x)=r(x)=x .
$$

Hence there is a point $x \in(A, \kappa)$ such that $h(x)=x$, where $h=r \circ f \circ i$. As a result, $(A, \kappa)$ has the fixed point property.

Proposition 3.12 Let $A, B$ be digital images with $\kappa$-adjacency. The $A \wedge B$ has the fixed point property if and only if both $A$ and $B$ have the fixed point property.

Proof Let $u$ be the common base point. For sufficient condition, suppose $f: A \wedge B \longrightarrow$ $A \wedge B$, and let us assume that $f(u) \in A$ and $f(u) \neq u$. Let $g: A \longrightarrow A$ be $p$, the projection of $A \wedge B$ onto $A$. Then $g\left(a_{0}\right)=a_{0}$ for some $a_{0} \in A$. If $f\left(a_{0}\right) \in A$, then

$$
a_{0}=g\left(a_{0}\right)=p \circ f\left(a_{0}\right)=f\left(a_{0}\right) \text {. }
$$


On the other hand, if $f\left(a_{0}\right) \in B$, then

$$
a_{0}=g\left(a_{0}\right)=p \circ f\left(a_{0}\right)=u
$$

contrary to the assumption that $f(u) \in A-B$. So, $f$ has the fixed point. Necessary condition follows from Theorem 3.11 .

The boundary $B d\left(I^{n+1}\right)$ of an $(n+1)$-cube $I^{n+1}$ is homeomorphic to $n$-sphere $S^{n}$. This allows us to represent a digital sphere by using the boundary of a digital cube. We use $0_{n}$ to denote the origin of $\mathbb{Z}^{n}$. Boxer [10] defines sphere-like digital image as follows:

$$
S_{n}=[-1,1]_{\mathbb{Z}}^{n+1} \backslash\left\{0_{n+1}\right\} \subset \mathbb{Z}^{n+1}
$$

Converse of the Lefschetz fixed point theorem need not be true for digital images. Let us see this.

Example 3.13 $S_{1}=\{(1,0),(1,-1),(1,1),(0,1),(0,-1),(-1,1),(-1,0),(-1,-1)\}$ is a digital 1 -sphere with 4-adjacency in $\mathbb{Z}^{2}$. It is the digital 4-closed curve $M S C_{4}$. By Example 2.10, we have

$$
H_{q}^{4}\left(S_{1}\right)= \begin{cases}\mathbb{Z}, & q=0,1 \\ 0, & q \neq 0,1\end{cases}
$$

Let $\phi:\left(S_{1}, 4\right) \longrightarrow\left(S_{1}, 4\right)$ be a digital $(4,4)$-continuous map. This map is defined as $\phi(z)=z$, where $z \in S_{1}$. The Lefschetz number of $\phi$ is

$$
\lambda(\phi)=\sum_{i=0}^{q} \operatorname{tr}\left(\phi_{i}: H_{i}^{4}\left(S_{1}\right) \longrightarrow H_{i}^{4}\left(S_{1}\right)\right)=1-1=0,
$$

since $\phi_{0}, \phi_{1}: \mathbb{Z} \longrightarrow \mathbb{Z}$ are the identity functions. Thus $\lambda(\phi)=0$, but $\phi$ has the fixed point. As a consequence, we have the following corollary.

Corollary 3.14 Converse of the Lefschetz fixed point theorem need not be true for digital images.

Now we compute the Lefschetz number of $S_{2}$. We define and sketch it.

Example 3.15 $S_{2}=[-1,1]_{\mathbb{Z}}^{3} \backslash\{(0,0,0)\}$ is a digital 2 -sphere with 6 -adjacency in $\mathbb{Z}^{3}$. It is the digital image $M S S_{6}$ in Example 2.11. Let $f:\left(S_{2}, 6\right) \longrightarrow\left(S_{2}, 6\right)$ be a digital $(6,6)$-continuous map. Using Theorem 3.2, the Lefschetz number of $f$ is

$$
\lambda(f)=\sum_{i=0}^{q} \operatorname{tr}\left(f_{*}: H_{i}^{6}\left(S_{2}\right) \longrightarrow H_{i}^{6}\left(S_{2}\right)\right)=1-23+0=-22,
$$

since $f_{0}: \mathbb{Z} \longrightarrow \mathbb{Z}$ is the identity function and $f_{1}: \mathbb{Z}^{23} \longrightarrow \mathbb{Z}^{23}$. As a result, $f$ has a fixed point. 
In [10], Boxer et al. defined the Euler characteristic of digital images. Let $(X, \kappa)$ be a digital image of dimension $m$, and for each $q \geq 0$, let $\alpha_{q}$ be the number of digital $(\kappa, q)$ simplexes in $X$. The Euler characteristic of $X$, denoted by $\chi(X, \kappa)$, is defined by

$$
\chi(X, \kappa)=\sum_{q=0}^{m}(-1)^{q} \alpha_{q}
$$

Moreover, they proved that if $(X, \kappa)$ is a digital image of dimension $m$, then

$$
\chi(X, \kappa)=\sum_{q=0}^{m}(-1)^{q} \operatorname{rank} H_{q}^{\kappa}(X)
$$

Proposition 3.16 Let $(X, \kappa)$ be a digital image. If a map $f:(X, \kappa) \longrightarrow(X, \kappa)$ is homotopic to the identity, then $\lambda(f)=\chi(X, \kappa)$.

Proof We could equally have defined the Lefschetz number without homology as follows:

$$
\lambda(f)=\sum_{i=0}^{n}(-1)^{i} \operatorname{tr}\left(f_{*}: H_{i}^{\kappa}(X) \longrightarrow H_{i}^{\kappa}(X)\right)=\sum_{i=0}^{n}(-1)^{i} \operatorname{tr}\left(f_{\#}\right),
$$

where $f_{\#}: C_{i}^{\kappa}(X) \longrightarrow C_{i}^{\kappa}(X)$. Since homotopic maps induce the same map on $n$-chains, we can consider $1_{\#}: C_{i}^{\kappa}(X) \longrightarrow C_{i}^{\kappa}(X)$. The map $1_{\#}$ is the $\alpha_{q}(X, \kappa) \times \alpha_{q}(X, \kappa)$ identity matrix and thus has the trace $\alpha_{q}(X, \kappa)$. As a result,

$$
\lambda(f)=\sum_{i=0}^{n}(-1)^{i} \operatorname{tr}\left(f_{\#}\right)=\sum_{i=0}^{n}(-1)^{i} \alpha_{i}(X, \kappa)=\chi(X, \kappa) .
$$

Example 3.17 Let $M S S_{6}^{\prime}$ in Example 2.12 be denoted by $(X, 6)$, and let $f:(X, 6) \longrightarrow(X, 6)$ be any $(6,6)$-continuous map. For $q>1, f_{q}: H_{q}^{6}(X) \longrightarrow H_{q}^{6}(X)$ is a zero map, $\operatorname{so} \operatorname{tr}\left(f_{q}\right)=0$. For $q=0, \operatorname{tr}\left(f_{*}\right)=1$ because $f_{0}$ is the identity function on $\mathbb{Z}$. On the other hand, for $q=1$, we get $\operatorname{tr}\left(f_{*}\right)=\operatorname{rank}\left(\mathbb{Z}^{5}\right)=5$ in that $5 \times 5$-matrix of $f_{1}: \mathbb{Z}^{5} \longrightarrow \mathbb{Z}^{5}$ is

$$
\left[\begin{array}{lllll}
1 & 0 & 0 & 0 & 0 \\
0 & 1 & 0 & 0 & 0 \\
0 & 0 & 1 & 0 & 0 \\
0 & 0 & 0 & 1 & 0 \\
0 & 0 & 0 & 0 & 1
\end{array}\right] .
$$

We have

$$
\lambda(f)=\sum_{i=0}^{3}(-1)^{i} \operatorname{tr}\left(f_{i}\right)=1-5+0=-4 \neq 0
$$

By Theorem 3.3, $f$ has a fixed point. The Euler characteristic of $M S S_{6}^{\prime}$ is $\chi\left(M S S_{6}^{\prime}, 6\right)=-4$.

We define a digital version of the real projective plane $\mathbb{R} P^{2}$ via a quotient map from $S_{2}$ with antipodal points. Denote the digital projective plane by $P^{2}$. We calculated the Lef- 


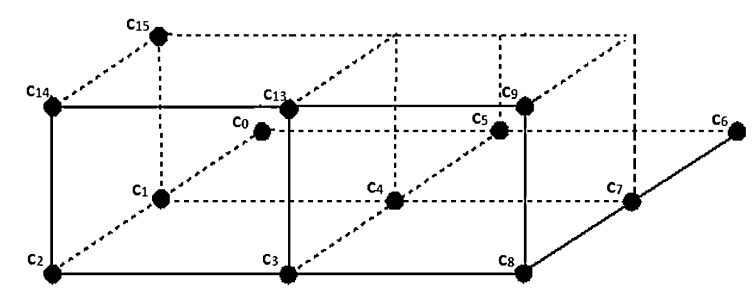

Figure 4 Digital projective plane $P^{2}$.

schetz number of $S_{2}$ in Example 3.15. Let

$$
\begin{aligned}
S_{2}= & \left\{c_{0}=(-1,-1,0), c_{1}=(0,-1,0), c_{2}=(1,-1,0), c_{3}=(1,0,0), c_{4}=(0,0,0),\right. \\
& c_{5}=(-1,0,0), c_{6}=(-1,1,0), c_{7}=(0,1,0), c_{8}=(1,1,0), c_{9}=(1,1,1), \\
& c_{10}=(0,1,1), c_{11}=(-1,1,1), c_{12}=(-1,0,1), c_{13}=(1,0,1), c_{14}=(1,-1,1), \\
& c_{15}=(0,-1,1), c_{16}=(-1,-1,1), c_{17}=(-1,-1,2), c_{18}=(0,-1,2), \\
& c_{19}=(1,-1,2), c_{20}=(1,0,2), c_{21}=(0,0,2), c_{22}=(-1,0,2), c_{23}=(-1,1,2), \\
& \left.c_{24}=(0,1,2), c_{25}=(1,1,2)\right\} .
\end{aligned}
$$

If we take the quotient map $q: S_{2} \longrightarrow S_{2} /_{x \sim-x}$, where $-x$ is the antipodal point of $x \in S_{2}$, then we have the digital projective plane $P^{2}$ (see Figure 4).

Define a map $H: P^{2} \times[0,4]_{\mathbb{Z}} \longrightarrow P^{2}$ by the following.

$$
\begin{cases}H(c, 0)=c, & t=0, \\ H\left(c_{15}, 1\right)=c_{14}, H\left(c_{0}, 1\right)=c_{5}, H\left(c_{1}, 1\right)=c_{4}, H\left(c_{2}, 1\right)=c_{3}, & t=1, \\ H\left(c_{14}, 2\right)=c_{13}, H\left(c_{5}, 2\right)=c_{6}, H\left(c_{4}, 2\right)=c_{7}, H\left(c_{3}, 2\right)=c_{8}, & t=2, \\ H\left(c_{13}, 3\right)=c_{9}, H\left(c_{6}, 3\right)=c_{7}, & t=3, \\ H\left(c_{7}, 4\right)=H\left(c_{9}, 4\right)=c_{8}, & t=4,\end{cases}
$$

where $c \in P^{2}$. It is clear that this map is a 6 -deformation retract of $P^{2}$. So, $P^{2}$ is 6-contractible image. As a result, $P^{2}$ has the same digital homology groups as a single point image. By Theorem 3.8, we have the following.

Corollary 3.18 Every $(6,6)$-continuous map $f: P^{2} \longrightarrow P^{2}$ has a fixed point.

\section{Conclusion}

The main goal of this study is to determine fixed point properties for a digital image. Moreover, we study the relations between the Euler characteristic and the Lefschetz number. At the end of this work, we give a digital version of the real projective plane and calculate its homology groups and the Lefschetz number. We expect that these properties will be useful for image processing. 
Authors' contributions

All the authors read and approved the final manuscript.

\section{Author details}

'Department of Mathematics, Celal Bayar University, Muradiye, Manisa 45140, Turkey. ${ }^{2}$ Departments of Mathematics, Ege University, Bornova, Izmir 35100, Turkey.

\section{Acknowledgements}

The authors thank the editor and the referees for their valuable suggestions to improve the quality of this paper

Received: 5 July 2013 Accepted: 30 September 2013 Published: 07 Nov 2013

\section{References}

1. Arslan, H, Karaca, I, Oztel, A: Homology groups of $n$-dimensional digital images XXI. Turkish National Mathematics Symposium, B1-13 (2008)

2. Boxer, L, Karaca, I, Oztel, A: Topological invariants in digital images. J. Math. Sci. Adv. Appl. 11(2), 109-140 (2011)

3. Demir, E, Karaca, I: Simplicial Homology Groups of Certain Digital Surfaces. Preprint (2012)

4. Karaca, I, Ege, O: Some results on simplicial homology groups of 2D digital images. Int. J. Inf. Comput. Sci. 1(8), 198-203 (2012)

5. Ege, O, Karaca, I: Fundamental properties of simplicial homology groups for digital images. Am. J. Comput. Technol. Appl. 1(2), 25-42 (2013)

6. Boxer, L: Homotopy properties of sphere-like digital images. J. Math. Imaging Vis. 24, 167-175 (2006)

7. Herman, GT: Oriented surfaces in digital spaces. CVGIP, Graph. Models Image Process. 55, 381-396 (1993)

8. Boxer, L: Digitally continuous functions. Pattern Recognit. Lett. 15, 833-839 (1994)

9. Boxer, L: A classical construction for the digital fundamental group. J. Math. Imaging Vis. 10, 51-62 (1999)

10. Boxer, L: Digital products, wedges and covering spaces. J. Math. Imaging Vis. 25, 169-171 (2006)

11. Boxer, L: Properties of digital homotopy. J. Math. Imaging Vis. 22, 19-26 (2005)

12. Spanier, E: Algebraic Topology. McGraw-Hill, New York (1966)

10.1186/1687-1812-2013-253

Cite this article as: Ege and Karaca: Lefschetz fixed point theorem for digital images. Fixed Point Theory and Applications 2013, 2013:253

\section{Submit your manuscript to a SpringerOpen ${ }^{\ominus}$ journal and benefit from:}

- Convenient online submission

- Rigorous peer review

- Immediate publication on acceptance

- Open access: articles freely available online

- High visibility within the field

- Retaining the copyright to your article 